ARTICLE

https://doi.org/10.1057/s41599-019-0330-x

\title{
Guns on social media: complex interpretations of gun images posted by Chicago youth
}

\author{
Desmond U. Patton ${ }^{1 \star}$, William R. Frey ${ }^{1} \&$ Michael Gaskell ${ }^{1}$
}

\begin{abstract}
How should we interpret gun images on social media? Take for example the shooting at Stoneman Douglas High School. Media articles revealed that the gunman, a white adolescent male, posted images of firearms and other weapons on his social media profile prior to the shooting. On the other hand, when it comes to Black communities, digital policing strategies often intercept images with guns and individuals thought to be associated with gangs before a crime is ever committed. In this study, we use a mixed methods approach, situated in social systems theory, to make meaning of gun posting behavior among Black youth who associate with gangs in Chicago. We collected and examined a corpus of Twitter images (1851) through snowball sampling of a well-known deceased gang member in Chicago and users in their Twitter network. We identified 560 images that contain guns and asked two distinct groups to annotate images: formerly gang-involved outreach workers, known as community domain experts, at a local Chicago violence prevention organization and Master of Social Work students at Columbia University. After comparing their results, findings highlighted the prevalence and frequency of gun image posting within this corpus and critical differences in how community domain experts and social work annotators perceive guns. The various underlying intents provide a rich source of knowledge for understanding the symbolic nature of guns in the digital age.
\end{abstract}

\footnotetext{
${ }^{1}$ Columbia School of Social Work, 1255 Amsterdam, New York, NY 10027, USA. *email: dp2787@columbia.edu
} 


\section{Introduction}

$\mathrm{n}$ recent years, social media has become an ecological environment or neighborhood (Stevens et al., 2017), where digital scripts and narratives convey a wide range of emotional attributes among them happiness, love, trauma, and pain. For young people living in communities with high rates of gun violence, social media becomes a digital street where taunts, arguments, and threats may play out over Twitter conversations or Facebook live feeds-the study of which elucidates how physical neighborhood conflicts unfold and who is involved (Lane, 2018). Recent research findings from the burgeoning cyberbanging literature indicate that using computational tools to identify handguns in Twitter communications is an important method of detecting signals of aggression from Black gang-involved youth in Chicago (Blandfort et al., 2019). However, when interpreting behavior from Black communities, the presence of guns may supersede the context in which an image exists. Thus some viewers may tend toward erroneous or inaccurate conclusions, weave them into a narrative, and potentially identify them as a proxy if building a case for premeditation in future legal proceedings (Patton et al., 2017).

While computational tools offer new ways of understanding the links between social media communication and gun violence, there are real concerns about the potential for misinterpreting images on social media in the absence of sufficient context. Interpreting gun images within a networked public comprised of highly visible social media content offers a unique set of challenges. Balancing real-world safety risks and the potential for mass surveillance and emerging technologies to reproduce existing inequities raises concerns regarding the "New Jim Code" (Benjamin, 2019). Focusing on youth living in communities with high rates of gun violence, Patton and colleagues (2017) argue that youth may discuss or post pictures of guns as a way to posture and display digital gravitas, rather than having real intent to carry out gun violence. While scholarly literature provides rich description of why persons may choose to carry guns (May, 1999; Watkins et al., 2008; Watts, 2019; Wilkinson and Fagan, 2001), little research examines when and why guns appear in social media posts. Community insights (e.g., backstories, language, and general context) into the reasons young people post gun images on social media also receive limited attention or acknowledgement in this literature.

This study examines the 2012-2017 gun image-posting behavior of Black youth-including some who self-identify as gang involved-who live in Chicago neighborhoods that have high rates of gun violence. We intentionally wrestle, and to some extent struggle, with the concern that online pictures of guns juxtapose racialized scripts and narratives that may frame how pictures of Black boys with guns are interpreted in the broader criminal justice context. We review extant literature on the relationship among guns, gangs, and social media, and then address three primary questions: the frequency, prevalence, and type of gun images posted among Black youth in Chicago; gun image-posting behaviors and the extent to which they are gendered; and a description and comparison of community domain experts' and social work annotators' perceptions of aggression in images with guns.

\section{Literature review}

The relationship between guns and gang-related homicides. In an examination of U.S. gun homicides between 1980 and 2008, Cooper and Smith (2012) found that gang-related homicides were more common than those committed as a result of interpersonal disputes or another type of crime. In contrast, of all non-gun homicides during the same period, gang homicides were less common than all other contexts. Gun deaths of gang-involved individuals compared to non-gang involved individuals in the United States are markedly disproportionate. The Geneva Small Arms Survey (2011) estimated that the murder rate for gang members was 893.4 per 100,000 residents, compared to 5.7 per 100,000 residents for the general population. Living in a neighborhood with high rates of violence increases the risk of joining a gang, exposure to violence, and becoming a victim of homicide (Merrin et al., 2015; Santilli et al., 2017). Furthermore living in such a neighborhood increases the likelihood of owning or having access to a gun (Roberto et al., 2018). Still, inquiry into the possession and use of firearms among gangs is a critically important area of public health research.

Though precise estimates of gun ownership among U.S. street gangs vary, surveys have consistently found that gang-involved individuals own firearms at a higher rate than their non-gangaffiliated peers (Bjerregaard and Lizotte, 1995; Decker et al., 1996; Wilkinson, 2003). Surveys of gang-involved and gang-affiliated people have yielded varied reasons for owning firearms. As mentioned, one common reason is to protect oneself and maintain a sense of personal safety while navigating dangerous neighborhoods (Wilkinson, 2003). Further, gang-involved individuals are more likely than non-gang involved individuals to engage in illegal behaviors (Alleyne and Wood, 2010; Klein and Maxson, 2010; O'Brien et al., 2013) and may more frequently encounter situations of heightened risk to personal safety (Wright et al., 1993). Symbolic aspects may also play a role in gun ownership, among them using a gun to establish status or to project a violent identity (Stretesky and Pogrebin, 2007). Ultimately, gun ownership or access represents a crucial factor in the prevention of gun violence.

The role of social media in gang violence. Pyrooz and colleagues (2015) interviewed current gang-involved individuals from five major U.S. cities and found a rate of internet use equal to that of their non-gang-involved peers. While there is some debate regarding whether gang-involved individuals use the internet for sophisticated criminal enterprises (e.g., phishing schemes) or instrumental activities (e.g., direct gang recruitment), there is evidence that this behavior is uncommon among U.S. street gangs (Pyrooz et al., 2015). There is, however, a tendency for ganginvolved individuals to engage in deviant and illegal online activities, such as illegally downloading music, and selling drugs (Pyrooz et al., 2015).

A growing area of concern among community groups and law enforcement is the practice of internet banging (Patton et al., 2013). Internet banging broadly includes online communications that promote gang affiliation, communicate threats, and share information about rivals to sympathetic local or national groups. These online communications, which are often inflammatory, disrespectful, and threatening toward rivals, have been blamed for inciting real-world violence (Patton et al., 2018a). Where gang conflict in the past may have escalated due to face-to-face encounters, the immediacy of online communications may result in increased conflict and escalation (Patton et al., 2018a). Patton and colleagues (2016a) interviewed individuals who work for community violence interruption groups and found that social media plays an increasing role in escalation of conflict between rival gangs. Outreach workers identified several behaviors that increased the likelihood of violent confrontation, including taunting (e.g., posting a video of oneself on another group's "turf"), disrespecting another group's symbols (e.g., throwing down or inverting a rival's hand sign), and disrespecting fallen rivals (Patton et al., 2013). 
While these digital practices certainly lead to conflict and increased intergroup tension, they also provide an opportunity. Community outreach workers can maintain awareness of the ongoing conflicts and issues that arise between individuals and groups. In some cases, outreach workers who have the trust and respect of the community can directly engage with individuals and groups through social media to work toward a resolution. Due to the volume of online communications and the nearly impossible task of monitoring all possible sources of conflict, efforts are being made toward developing machine-learning tools to automatically identify predictors of online violence to render community outreach efforts more effective (Blandfort et al., 2019; Blevins et al., 2016; Chang et al., 2018).

The specific online behaviors that predict real-world violence are not fully understood, though qualitative analysis of ganginvolved youth's Twitter communications reveals aggressive themes as a potential source of escalation between groups (Patton et al., 2013). Loss and grief likely also play a role: there is a welldescribed cycle of violence in which gang murders occur in retaliation for a previous murder. Evidence of loss-related tweets predicting aggression-related tweets further suggests the importance of understanding expressions of loss as a potential antecedent to gun violence (Patton et al., 2018b). Aside from identifying real-time conflict, community, and law enforcement gang violence prevention strategies encompass a broad range of strategies including preventing initial gang involvement, suppressing gang activities, and using criminal justice strategies (Gravel et al., 2013). A recent report suggests that targeting areas with high rates of violence and removing illegally possessed firearms is also an effective strategy in reducing gang-associated gun violence (Webster et al., 2018). However, strategies that overlook systemic issues impacting who experiences gun violence may overlook a potential relationship between root causes of violence and gun violence prevention strategies. In the next section, we use social systems theory to better understand how sociohistorical influences may shape current gun posting behaviors on Twitter.

Social systems and blackness. Understanding gun image-posting behaviors of Black youth who live in neighborhoods with high rates of gun violence requires critical consideration of the social systems in which these behaviors take place. Without considering social systems and the sociohistorical ways in which Blackness is criminalized and perceived as a constant threat, social media interpretations can and have reinforced racist, reductionist explanations, and narratives to explain Black youth's social media behaviors (Lane, 2018). Crawford and Calo's (2016) socialsystems approach to artificial intelligence (AI) development and research describes a process that asks questions about the impact and effect of systems on both people and AI. Expanding this approach beyond AI systems provides a framework for examining the impact of harmful behavioral classification of the social media posts of Black youth. These youth in particular often experience compounded systematic marginalization, and their lived experiences are often extracted and decoded using AI systems.

A social-systems approach requires expansive inquiry and questions that take into account the systems and processes influencing Black youth behaviors. This approach also focuses on humanizing people who are often dehumanized, especially young people who may be gang-involved or affiliated (Frey, 2018). Rather than solely considering personal reasons for posting images of guns on social media, the social-systems approach pushes us to understand how systems of violence could influence pressures to display a tough persona online. Further, this approach allows researchers to prioritize ending gun violence, while carefully navigating concerns such as new technologies used to enforce the New Jim Code (Benjamin, 2019); further embedding surveillance capitalism in the fabric of society (Zuboff, 2019); and enhancing the sociopolitical and historical surveillance of Blackness (Browne, 2015). As Browne (2015) states, "How is the frame necessarily reframed by centering conditions of blackness when we theorize surveillance?" Otherwise put, how do our inquiries shift when we contextualize race as a sociopolitical system that leads to marginalization and hypersurveillance of Black communities? These questions must be central to any analysis of social media posting behaviors of Black youth. We must ask what various social systems impact Black youth who post images of guns on their own (at times, identifiable) social media profiles?

Extending the social-systems approach, Frey and colleagues (2018) identified the necessity of involving community/domain experts in research processes as well as the development and oversight of computational systems. There are limitations to the efficacy of employing a social-systems approach focused on broader questions and furthering our own understandings of gun images on social media, unless we holistically involve, at every stage in the process, the people being studied.

\section{The current study}

In this study, we examine three broad topics: (1) the prevalence of gun images in, and frequency with they appear, in our Twitter corpus; (2) gun image-posting behaviors on social media among gang-involved or gang-affiliated individuals in Chicago; (3) and how the presence of guns in images influence community domain experts and social work annotators' the perception of aggression. We conclude by wrestling with how guns are interpreted by situating our work in sociological research that examines how offline behavior, symbolism, and impressions shape digital posting practices in an ecology of violence.

\section{Methods}

Sampling and data collection. The data used in these analyses come from a broader research effort related to online communications among young people from marginalized communities in Chicago. The sample contains tweets collected in two phases. Table 1 provides a summary of the two resulting datasets.

The primary dataset is comprised of publicly available tweets for 274 individuals believed to be gang-involved or gang-affiliated in Chicago. To obtain 274 users, we used a snowball sampling technique-a commonly used method in sociological research when recruiting members of a group through random sampling is unfeasible (Atkinson and Flint, 2001). Our seed user was female, a well-known Chicago gang member, and a prolific Twitter user who was killed in April 2014. We added to the sample Twitter users who were top communicators with the seed user and those who were top communicators with them. In February 2017, we collected each of the 274 users' previous 200 tweets for a total sample of 52,053 tweets. Due to the infrequency of tweeting among some users, the earliest tweets came from as early as October 2012; however, $85 \%$ of tweets fell within one year of the sampling date, and $50 \%$ within a month of the sampling date.

The second phase of data collection involved sampling 20 additional publicly available original content tweets with images per user for an original user subset of 167. After removing duplicates from the primary dataset, this second phase resulted in 1856 additional tweets ranging from July 2013 to February 2017. Over $90 \%$ of the secondary dataset tweets originated within one year of the date on which data was obtained; approximately one quarter of tweets originated within one month of the sample date. Our interest was the behavior of displaying gun images online, we filtered the primary dataset to remove tweets without images. 


\section{Table 1 Descriptive summary of twitter datasets}

\begin{tabular}{lll} 
& Primary & Secondary \\
\hline Type & $\begin{array}{l}\text { Original and retweeted content, tweets with } \\
\text { images, text-only tweets }\end{array}$ & Original content, tweets with images \\
Number of users & $\begin{array}{l}\text { 274; Snowball sample from gang-involved, } \\
\text { well-known seed user }\end{array}$ & 167; Curated subset of primary dataset, most recently active users \\
Total tweets sampled & 52,053 & 1856 \\
Total image tweets & 4104 & 1856 \\
Inaccessible image tweets & 576 & 446 \\
Accessible image tweets with gun & 392 & 169 \\
in any context & 184 & 169 \\
Original gun images & 208 & 0 \\
Retweeted gun images & Overall frequency, gun image description, & Gun images description, thematic frequencies, comparison of \\
Analysis & thematic frequencies & academic and local domain expert thematic perceptions \\
\end{tabular}

This resulted in 4104 tweets with images which, combined with 1856 image tweets from the secondary dataset, provided a total of 5960 image tweets.

Due to the dynamic nature of social media data, user profiles, tweets, and images became unavailable over the course of the coding process. The reasons for unavailability were not always immediately known, but causes included changes to usernames, changes to privacy settings, suspension of profiles, and deletion of profiles. By the end of data analysis, 576 of the primary image tweets and 446 of the secondary image tweets were no longer available. Missing data due to unavailability of profiles is described whenever applicable throughout the results. To protect the identities and privacy of Black youth in our corpus, we do not include images in this paper (Tweet IDs for the data may be made available to researchers who sign a memorandum of understanding specifying their intended use of the data and their agreement with our ethical guidelines).

Image coding. The authors coded all image tweets for the presence of a firearm in any context. We then coded all firearm images on concrete variables of interest related to the image (e.g., number of guns, size of magazine, etc.), which we describe in more detail below. Thematic codes for the two datasets follow the convention of previous research within this population and include four categories: aggression, loss, substance use, and "other" (Blevins et al., 2016; Patton et al., 2016a). We applied thematic codes through an intensive, contextually driven annotation process. Next, two social work student annotators, who had more than $100 \mathrm{~h}$ of coding and labeling experience in this broader dataset, each provided thematic codes for all images in the secondary dataset. Disagreements were reconciled through a reconciliation process with a senior member of the research team. For gun image tweets not included in the secondary dataset, the senior researcher provided a thematic code.

Additionally, all image tweets from the secondary dataset were coded by domain experts from the local community. The domain experts were African American, one man and one woman in their early to mid-twenties. Both domain experts were employed as outreach workers at a local Chicago violence-prevention nonprofit organization. The domain experts were selected because of past experience with gun violence, use of social media as an outreach tool, and general interest in the research study. Domain experts coded social media content based on their lived experience, gun-violence expertize, and social media behavior of youth in their community. The domain experts did not receive the same training as social work annotators, or our analysis methods, because their lived experience was viewed as ground truth and true expertize (Frey et al., 2018).
We include domain experts from local communities in our work to help improve coding accuracy and minimize bias (Frey et al., 2018). The parallel coding process (lab annotators and domain experts) allows an exploratory analysis of the impact of gun presence in tweets. It also allows a comparison between perceptions of lab annotators who-despite a deeper-than-average understanding of the target population-do not live in the context of the tweets and community-based domain experts, who live in the context and have a deep and nuanced understanding of places, people, and things in the images. Due to the non-experimental nature of this dual coding process, these findings will be treated similar to a case study and only potential trends will be discussed.

Variables. Tweets were coded on three domains: general aspects of the tweet or image that did not pertain directly to the firearm; attributes and behaviors regarding the pictured firearms; and the thematic content of the tweets.

General tweet attributes. We coded tweets on several general attributes including the date of the tweet, whether the tweet was original content or a retweet, and the type of image depicted (e.g. photograph, meme, etc.). To explore gender-based differences in behaviors, we also coded each tweet according to the tweet author's apparent gender. Since gender is usually not explicitly stated on Twitter, we determined gender was based on stereotypical hairstyles, clothing, and pronouns used. We only coded images on this variable when the gender of the user could be determined with high confidence.

Gun attributes and gun behaviors. Precise classification of firearms is difficult, given the absence of a clear classification system. As such, we coded firearms as simply semi-automatic, revolver, and rifle. We also coded the size of the handgun frames as either standard or compact. Since there is disagreement regarding what constitutes a full-sized versus compact pistol frame, handguns were usually considered full-sized unless a markedly small frame was observed. However, in instances where the brand and model name were visible, the frame's designation in the company's marketing materials was used to classify the firearm as standard or compact. For magazine-fed handguns, magazine type was determined as standard, extended, or drum. The convention used for classifying magazines set standard magazines as flush or within two finger-widths of the bottom of the pistol grip, extended magazines as straight and extending beyond this length, and drum magazines as containing one or two cylindrical feeding mechanisms. The specific behavior depicted with the gun was also coded. Categorical codes were developed through qualitative analysis of a subset of the data and are described in more detail 
Table 2 Gender differences within the dataset

\begin{tabular}{|c|c|c|c|c|c|c|c|c|}
\hline & Men & Women & $x^{2}$ & Df & $\boldsymbol{p}$ & Unavailable & Undetermined & Total \\
\hline Entire sample & $155(53.3 \%)$ & $54(18.6 \%)$ & 48.809 & 1 & $<0.001$ & $67(29 \%)$ & $15(5.2 \%)$ & 291 \\
\hline Valid \% & 74.2 & 25.8 & & & & & & \\
\hline Users who post guns & $92(57.5 \%)$ & $19(11.8 \%)$ & 4.442 & 1 & $<0.05$ & $38(23.8 \%)$ & $11(6.9 \%)$ & 160 \\
\hline Valid \% & 82.9 & 17.1 & & & & & & \\
\hline Original Gun images & $75(62.5 \%)$ & $11(9.2 \%)$ & 7.617 & 1 & $<0.01$ & $28(23.3 \%)$ & $6(5 \%)$ & 120 \\
\hline Valid \% & 87.2 & 12.8 & & & & & & \\
\hline Guns IRL & $57(70.4 \%)$ & $4(4.9 \%)$ & 11.741 & 1 & $<0.001$ & $19(23.5)$ & $1(1.2 \%)$ & 81 \\
\hline Valid \% & 93.4 & 6.6 & & & & & & \\
\hline
\end{tabular}

below. Additionally, specific behavior of covering or obscuring one's face was also tabulated.

Actual possession. We also recognized the importance of identifying images that suggest the tweet author or a close acquaintance presently owns or has immediate access to a gun. As compared to images that do not reflect this circumstance, actual possession images are of greater importance to individuals who are actively attempting to prevent gun violence and likely influence the perceived threat of an image. Images that met these criteria but were clearly historical photos did not qualify for this code.

\section{Thematic content}

\section{Aggression}

The aggression thematic code encompasses expressions of insults, taunts, threats, or other communications that suggest possible aggression toward another person or group.

\section{Loss}

This code includes communication that references feelings of grief, sadness, or sorrow in relation to the loss of a loved one due to death or incarceration. Loss also includes communications related to the ongoing remembrance or memorialization of a deceased friend or loved one.

\section{Substance use}

The substance use thematic code includes the presence of a substance other than cigarettes in an image coupled with some indication of current or future use.

\section{Other}

Communications related to any matter not otherwise covered by the previous themessports, music, food, relationships, daily life-were categorized as "other."

\section{Results}

Frequency and prevalence of gun images. Of the 3528 available tweets with images contained in the primary dataset, $392(11.1 \%)$ depicted a firearm in any context. Of these, 184 (47.0\%) were original-content gun images and 208 (53\%) were retweeted images. In comparison to the overall sample, images containing guns were more likely to be original content than retweets $\left(c^{2}[1\right.$, $N=392]=20.36, p<0.001)$. These images were posted by 119 (43.4\%) of the 274 unique Twitter handles from the primary dataset. The average number of gun images per unique user was $3.29(\mathrm{SD}=3.54)$. Of the available 1410 images in the secondary dataset, $169(12.0 \%)$ depicted a gun in any context. These images were posted by 86 of the 173 (49.7\%) unique Twitter handles. The average number of unique posts per Twitter handle was 1.97 $(\mathrm{SD}=1.43)$. Across the entire sample, $40.5 \%$ of gun images depicted a gun in actual possession. In $36.4 \%$ of images, there was strong evidence that the gun depicted was not in actual possession. In $18.2 \%$ of cases, a gun in actual possession was plausible, but insufficient information precluded a definitive determination. In $4.8 \%$ of cases, missing data precluded a determination.
Perceived gender. Gender differences within the sample are presented in Table 2.

Across the available users in both samples, male accounts $(n=155)$ were more common than female accounts $(n=54)\left[c^{2}\right.$ $(1, N=209]=48.81, p<0.001)$. We conducted chi square tests to determine whether gender differences differed from the expected values given the gender proportions of the total dataset. The findings for users who posted at least one gun image in any context, users who posted an original content gun image, and users who posted an image depicting actual possession are reported in Table 2. The number of men exceeded the hypothesized value across all gun-image posting groups, and men were increasingly over-represented as the subjective gravity of the type of image posted increased.

Gun attributes. On average, each image in our Twitter corpus depicted $1.65(\mathrm{SD}=1.39)$ firearms. Semi-automatic handguns were the most common type of firearm representing $91.7 \%$ of all guns depicted. Revolvers and rifles represented $3.8 \%$ and $3.6 \%$, respectively. For $3.0 \%$ of the images, the image had insufficient detail (e.g. picture was fuzzy or unclear) to determine the type of gun. Of semi-automatic handgun magazines, standard capacity magazines were used in $62.7 \%$ of the guns depicted, extended magazines in $34.6 \%$, and drum-style magazines in $2.8 \%$.

Gun image-posting behavior. Several behaviors associated with the position of the firearm were identified across the images. The most common behavior depicted pointing the gun at the camera, which accounted for $29.7 \%$ of the guns depicted. The second most-frequent behavior $(20.2 \%)$ was holding the gun in a ready position, or in a manner that reflected relaxed stance. This behavior almost always depicted the individual holding the firearm with his or her finger off the trigger and not pointed in the direction of another person-two common rules of firearm safety.

In contrast, $17.9 \%$ of guns were held in an atypical fashion, and the gun was not held in a ready position. For example, in some images the firearm was held upside down by the end of the magazine; in others, guns were not held at all by the user (16.4\%), such as when a gun was depicted lying on a table. In $7.1 \%$ of images, guns were depicted on one's person. Most often, guns were kept in a waistband or carried around the neck on a lanyard. No holsters were depicted in the images. On occasion, guns were depicted as being pointed or placed in a way that had symbolic meaning. For example, one hand might hold a gun against one's temple while the other hand covers the mouth-a symbolic communication apparently referencing speaking with police or "snitching."

Visibility of faces. The vast majority $(74.5 \%)$ of images included the subject's face in full view. In $9.8 \%$ of images, at least one face in the image was obscured. In many images, plumes of smoke or low lighting reduced the level of detail of an individual's face to 

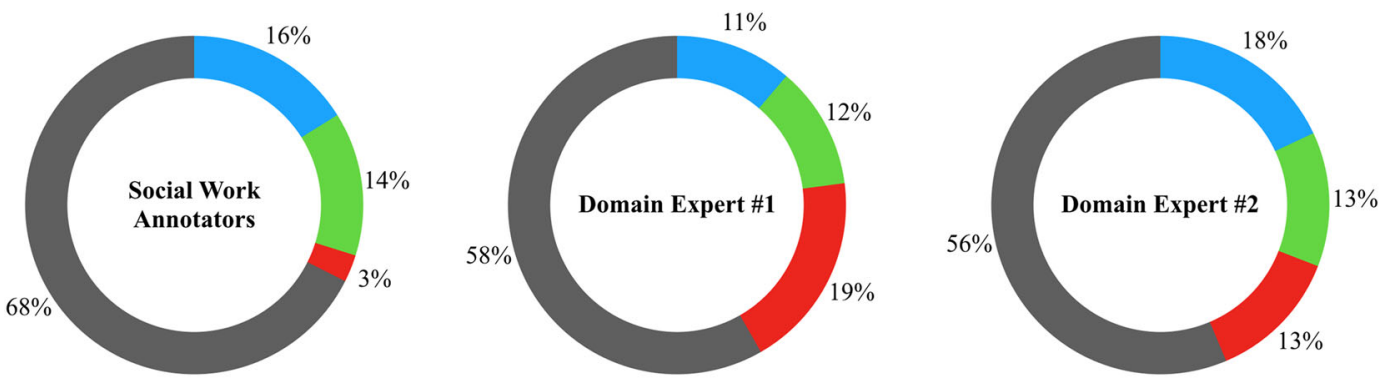

Loss

Substance Use

Aggression

Other

Fig. 1 Thematic code breakdown for all secondary image tweets

\begin{tabular}{|c|c|c|c|c|}
\hline & Odds ratio & $95 \% \mathrm{Cl}$ & $\boldsymbol{Z}$ & $p$ \\
\hline Domain Expert \#1 & 20.7090 & $13.5869-31.5644$ & 14.094 & $<0.0001$ \\
\hline Domain Expert \#2 & 17.8550 & $12.1009-26.3454$ & 14.522 & $<0.0001$ \\
\hline S.W. Annotators & 16.2478 & 8.7106-30.3070 & 8.765 & $<0.0001$ \\
\hline
\end{tabular}

the point that face identification was unlikely. In another $12 \%$ of images, faces were not featured in the image, for example when a firearm is pictured at someone's feet or lying on a table. Though these do not demonstrate a direct attempt to obscure identity, they may or may not represent the subject's effort to avoid having his or her face in an image. Subjects in just $3.6 \%$ of images made a clear attempt to obscure their identity. This was most often done using a mask, though in some instances, faces were blocked by an overlaid emoji.

Thematic content. Social work annotators and domain experts from Chicago categorized the entire secondary dataset. In Fig. 1 we present the thematic code proportions across social work and domain expert annotators for all 1687 image tweets from our dataset. In general, community domain experts categorized image tweets as aggressive at a rate of five to seven times that of lab annotators. Odds ratios were calculated to determine how the presence of a gun affected the likelihood of categorizing a tweet as aggressive (Table 3).

Across both domain experts and social work annotators, the presence of a gun greatly increased the likelihood that an image tweet would be coded as aggressive. Two general trends emerged while comparing these data. First, though social work annotators were less likely to see aggression in general, this effect was more pronounced when a gun was not present in an image. In this situation, just $1.3 \%$ of images were considered aggressive by annotators, compared to $8.4 \%$ and $13.7 \%$ of images considered aggressive by domain experts. Second, when a gun was present, annotators made lower estimates of aggression, but they were closer to the thematic coding levels of domain experts.

Discrepancies in non-gun aggression labeling. Interestingly, social work annotators saw less aggression than domain experts did when there was no gun present in the image-the inverse of the previous theme. When a gun is not present in an image, annotators rely on less-obvious cues of aggression. Through follow-up interviews with domain experts, we discovered several indicators of aggression that had not been understood or captured by social work annotators. These included the reputation of the person in the image, specific aspects of local gang hand signs, and subtle language-based signals. Alternatively, differences in interpretation between domain-expert and social-work annotators may be explained by the different processes they used to code the Twitter data. Social work annotators used a seven-step analysis process designed to support reviewers in fully grasping the meaning of a tweet. This process is slow, requiring social work annotators up to 2 weeks to analyze 100 tweets. In contrast, domain experts completed the coding task in isolation and without using the CASM process. As such, they coded the image tweets quickly (100 tweets coded in this manner in 1-2 h). At times, this may have produced a more surface-level analysis of the tweets. From a cognitive perspective, coders may have activated more automatic schemas that led to an over-reliance on aggressive cues.

\section{Discussion}

Given the prevalence of gang-associated gun violence and the role of social media in understanding conflict and preventing inperson confrontation, analysis of our findings yielded insight into two related areas of inquiry: factors contributing to gun imageposting behavior and the complexity associated with accurate interpretation of gun image communication and its potential impact.

Factors contributing to gun image-posting behavior. In reviewing their gun image-posting behavior, we find that although images of a gun, or sometimes multiple guns, appeared frequently in their Twitter images, their posting behavior-how they held the guns, and the visibility of their faces-paints a more complex story. The layers of posting behavior, together with the nuanced cultural, racial, ecological, and developmental factors that shape social media engagement among Black youth (Patton et al., 2013) make it difficult to interpret the images and determine the intent of the gun.

The frequency of gun images in any context in this group is about $11 \%$. In other words, $\sim 1$ in 10 images posted by the sampled population contained an image of a gun. Factors contributing to frequent gun image-posting behavior among the sampled population can be usefully approached via a symbolic interactionism framework. Blumer (1986) posits that "human interaction is mediated by the use of symbols and by interpretation, or by ascertaining of one anothers' actions" (p. 180). Wilkinson and Fagan (1996) argue that the presence of guns, in an offline context, influences how decisions are made in both the social interactions that lead to disputes and in ongoing disputes. Interpretation of gun-image posting and reaction to guns is couched in a broader social context: Offline, firearm injuries are concentrated in urban communities of color that also experience extreme levels of resource deprivation (Wilkinson and Fagan, 1996). 
In a social media environment, gun image-posting may be influenced by street codes and an ecology of danger. Anderson (2000) notes that street orientations govern the normative systems regarding human behavior in public space. Lane (2018) advanced this theory by suggesting that street codes are filtered through digital technology. More specifically, neighborhood risks and opportunities associated with urban poverty are mediated through the use of popular social networking tools like Twitter. Thus, images that depict an individual holding a firearm with his or her finger off the trigger and not pointed in the direction of another person, or images in which the subject displays his or her face while holding a gun, may be associated with digital street scripts. These behaviors aim to achieve and further social identity, convincing others to believe that identity, rather than conveying actual intent to use the firearm in the image.

Wilkinson and Fagan (1996) suggest that the importance of stature and reputation, as conveyed through posting a gun image on Twitter, may influence how an individual is perceived online and potentially confronted offline. Analysis of our datasets found that posted gun images depicted a variety of behaviors, but that it was quite common for guns to be held and pointed at the camera, in a "ready" position, or held in an atypical manner. Less commonly, guns were not held or were held in a symbolic way. A small subset of guns were depicted as stored on one's person. Most commonly guns stored on a person were tucked into a waistband. In general, gun-holding behaviors seemed to reflect a desire to display the gun, as opposed to the incidental capturing of daily firearm carrying behaviors. In addition, the vast majority of gun images are of semi-automatic weapons, though some revolvers and rifles were observed.

Following this analysis, one might conclude that this specific type of gun posting behavior aims for impression management. Among the sampled population, posting of gun images appears to focus on the visibility of the gun as a digital street script. These scripts may articulate one's bravado, toughness, or protection strategy.

The complexity of accurately interpreting gun images. In this study, domain experts lived in the same and similar communities as the Twitter users sampled. Research suggests that young people use social media to navigate their violent neighborhoods (Patton et al., 2013). Because young people use social media to navigate their neighborhoods, it may serve them if others interpret aggression from their online communications. The reality is, while a false positive (e.g. identifying text as aggression) may result in increased caution for no reason, a false negative (e.g. not identifying text as aggression) could lead to death. In addition, domain experts have a deep, nuanced knowledge of the culture, people, and places required to understand a social media post in context (Frey et al., 2018). While domain experts regarded most gun images as aggressive, a significant portion ( $23 \%$ for one domain expert and $40 \%$ for the other) of image tweets were not considered aggressive.

Interestingly, expressions of loss were the next most common theme in gun images. The frequency of this co-occurrence suggests an interesting link between aggression and loss and how they are perceived and communicated on social media (Patton et al., 2018b).

It is possible that images with guns are more likely to be labeled as aggressive because the gun is a salient symbol of aggression. An ecology of danger framing might suggest that social interactions perceived as threatening or lethal and the associated individuals are normatively seen as harboring hostile intent or the willingness to inflict harm (Wilkinson and Fagan, 1996). The welldocumented cognitive and behavioral effect of being exposed to gun imagery, or "weapons effect," suggests that when we see a gun or an image of a gun, we become primed to think and behave aggressively (Berkowitz and LePage, 1967). This effect may even be present when the gun is an image and not in a real-life context.

\section{Conclusion}

Sensitive and accurate interpretation of the meaning of gun images in tweets posted by Black Chicago youth is a complex undertaking. While gun images are frequent and pervasive, prior literature and a social-systems approach warn against drawing punitive and potentially harmful conclusions without a humanistic and contextually informed understanding of the broad, diverse, and complicated reasons posting gun images on Twitter may serve Black Chicago youth. However, this study is not without limitations. It relies on the interpretations of gun images from violence outreach workers and master of social work students. While both groups have domain knowledge that is culturally nuanced, specific, and based on their lived experience, including the perspectives of individuals posting the images, individuals in the images, friends in their networks, and police officers who may interpret the images could enhance this work.

This study is an important first step in a critical assessment of how we interpret guns on social media and how we can leverage distinct populations' interpretations to uncover potential bias and gaps in understanding the meaning of gun images on social media - particularly those images posted by members of marginalized Black communities. When not used carefully, careless interpretations of gun images on social media can be, and have been, used as evidence to target individuals for arrest, as seen in New York City's Operation Crew Cut efforts (Lane, 2018). At the same time, posting images of guns online may influence online and offline conflict. Future research should further investigate the role of gun imagery and social media as a means of identifying conflicts and preventing violence.

\section{Data availability}

The dataset generated and/or analyzed during the current study is not publicly available due to the sensitive nature of the content, but are available from the corresponding author on reasonable request and signing of a MOU to ensure the ethical use of data.

Received: 2 August 2019; Accepted: 16 September 2019; Published online: 15 October 2019

\section{References}

Alleyne E, Wood J (2010) Gang involvement: psychological and behavioral characteristics of gang members, peripheral youth, and nongang youth. Aggressive Behav 36(6):423-436

Anderson E (2000) Code of the street: decency, violence, and the moral life of the inner city. WW Norton \& Company

Atkinson R, Flint J (2001) Accessing hidden and hard-to-reach populations: Snowball research strategies. Soc Res Update 33(1):1-4

Benjamin R (2019) Race after technology: Abolitionist tools for the new jim code. John Wiley \& Sons

Berkowitz L, LePage A (1967) Weapons as aggression-eliciting stimuli. J Personal Soc Psychol 7(2, Part 1):202-227

Bjerregaard B, Lizotte AJ (1995) Gun ownership and gang membership. J Crim law Criminol 86(1):37-58

Blandfort P, Patton DU, Frey WR, Karaman S, Bhargava S, Lee FT, Varia S, Kedzie C, Gaskell MB, Schifanella R, McKeown K (2019) Multimodal social media analysis for gang violence prevention. In Proceedings of the International AAAI conference on web and social media, vol 13(1). pp. 114-124

Blevins T, Kwiatkowski R, Macbeth J, McKeown K, Patton D, Rambow O (2016) Automatically processing tweets from gang-involved youth: towards detecting loss and aggression. In Proceedings of the COLING 2016, the 26th international conference on computational linguistics: technical papers. pp. 2196-2206 
Blumer H (1986) Symbolic interactionism: perspective and method. University of California Press

Browne S (2015) Dark matters: on the surveillance of blackness. Duke University Press

Chang S, Zhong R, Adams E, Lee FT, Varia S, Patton D, Frey W, Kedzie C, McKeown K (2018) Detecting gang-involved escalation on social media using context. In Proceedings of the 2018 conference on empirical methods in natural language processing. pp. $46-56$

Cooper A, Smith EL (2012) Homicide trends in the United States, 1980-2008. BiblioGov

Crawford K, Calo R (2016) There is a blind spot in AI research. Nature 538 (7625):311-313

Decker S, Decker SH, Van Winkle B (1996) Life in the gang: family, friends, and violence. Cambridge University Press

Frey WR (2018) Humanizing digital mental health through social media: centering experiences of gang-involved youth exposed to high rates of violence. Biomed Inform Insights 10:1178222618797076

Frey WR, Patton DU, Gaskell MB, McGregor KA (2018) Artificial intelligence and inclusion: formerly gang-involved youth as domain experts for analyzing unstructured twitter data. Soc Sci Comput Rev https://doi.org/10.1177/ 0894439318788314

Geneva Small Arms Survey (2011) Small arms survey 2011: states of security. Cambridge University Press

Gravel J, Bouchard M, Descormiers K, Wong JS, Morselli C (2013) Keeping promises: a systematic review and a new classification of gang control strategies. J Crim Justice 41(4):228-242

Klein MW, Maxson CL (2010) Street gang patterns and policies. Oxford University Press

Lane J (2018) The digital street. Oxford University Press

May DC (1999) Scared kids, unattached kids, or peer pressure: why do students carry firearms to school? Youth Soc 31(1):100-127

Merrin GJ, Hong JS, Espelage DL (2015) Are the risk and protective factors similar for gang-involved, pressured-to-join, and non-gang-involved youth? A socialecological analysis. Am J Orthopsychiatry 85(6):522-535

O'Brien K, Daffern M, Chu CM, Thomas SD (2013) Youth gang affiliation, violence, and criminal activities: a review of motivational, risk, and protective factors. Aggression Violent Behav 18(4):417-425

Patton DU, Brunton DW, Dixon A, Miller RJ, Leonard P, Hackman R (2017) Stop and frisk online: theorizing everyday racism in digital policing in the use of social media for identification of criminal conduct and associations. Soc Media + Soc 3(3):2056305117733344

Patton DU, Eschmann RD, Butler DA (2013) Internet banging: new trends in social media, gang violence, masculinity and hip hop. Comput Hum Behav 29(5): A54-A59

Patton DU, Eschmann RD, Elsaesser C, Bocanegra E (2016a) Sticks, stones and Facebook accounts: what violence outreach workers know about social media and urban-based gang violence in Chicago. Comput Hum Behav 65:591-600

Patton DU, McGregor K, Slutkin G (2018a) Youth gun violence prevention in a digital age. Pediatrics 141(4):e20172438

Patton DU, Rambow O, Auerbach J, Li K, Frey W (2018b) Expressions of loss predict aggressive comments on Twitter among gang-involved youth in Chicago. npj Digit Med 1(1):11

Pyrooz DC, Decker SH, Moule Jr RK (2015) Criminal and routine activities in online settings: gangs, offenders, and the Internet. Justice Q 32(3):471-499

Roberto E, Braga AA, Papachristos AV (2018) Closer to guns: the role of street gangs in facilitating access to illegal firearms. J Urban Health 95(3):372-382

Santilli A, O'Connor Duffany K, Carroll-Scott A, Thomas J, Greene A, Arora A, Agnoli A, Gan G, Ickovics J (2017) Bridging the response to mass shootings and urban violence: exposure to violence in New Haven, Connecticut. Am J Public Health 107(3):374-379
Stevens R, Gilliard-Matthews S, Dunaev J, Woods MK, Brawner BM (2017) The digital hood: social media use among youth in disadvantaged neighborhoods. New Media Soc 19(6):950-967

Stretesky PB, Pogrebin MR (2007) Gang-related gun violence: socialization, identity, and self. J Contemp Ethnogr 36(1):85-114

Watkins AM, Huebner BM, Decker SH (2008) Patterns of gun acquisition, carrying, and use among juvenile and adult arrestees: evidence from a highcrime city. Justice Q 25(4):674-700

Watts SJ (2019) Gun carrying and gun victimization among American adolescents: a fresh look at a nationally representative sample. Vict Offenders 14 (1):1-14

Webster DW, Buggs SA, Crifasi CK (2018) Estimating the effects of law enforcement and public health interventions intended to reduce gun violence in Baltimore. John Hopkins Bloomberg School of Public Health, Baltimore, MD, USA

Wilkinson DL (2003) Guns, violence, and identity among African American and Latino youth. LFB Scholarly Publishing

Wilkinson DL, Fagan J (1996) The role of firearms in violence scripts: the dynamics of gun events among adolescent males. Law Contemp Probs 59:55-89

Wilkinson DL, Fagan J (2001) What we know about gun use among adolescents. Clin Child Fam Psychol Rev 4(2):109-132

Wright JD, Sheley JF, Smith MD (1993) Kids, guns, and killing fields. Society 30 (1):84-89

Zuboff S (2019) The age of surveillance capitalism: the fight for a human future at the new frontier of power. Profile Books

\section{Acknowledgements}

The authors would like to thank the Institute for Nonviolence Chicago for their thoughtful and important contributions to this article.

\section{Competing interests}

The authors declare no competing interests.

\section{Additional information}

Correspondence and requests for materials should be addressed to D.U.P.

Reprints and permission information is available online at http://www.nature.com/ reprints

Publisher's note Springer Nature remains neutral with regard to jurisdictional claims in published maps and institutional affiliations.

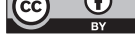

Open Access This article is licensed under a Creative Commons Attribution 4.0 International License, which permits use, sharing, adaptation, distribution and reproduction in any medium or format, as long as you give appropriate credit to the original author(s) and the source, provide a link to the Creative Commons license, and indicate if changes were made. The images or other third party material in this article are included in the article's Creative Commons license, unless indicated otherwise in a credit line to the material. If material is not included in the article's Creative Commons license and your intended use is not permitted by statutory regulation or exceeds the permitted use, you will need to obtain permission directly from the copyright holder. To view a copy of this license, visit http://creativecommons.org/ licenses/by/4.0/

(c) The Author(s) 2019 